\title{
THE NUCLEAR LENGTH OF A CHARACTER
}

\author{
WAYNE W. WHEELER
}

(Received 10 September 1993)

Communicated by L. G. Kovács

\begin{abstract}
If $G$ is a $\pi$-separable group and $\chi$ is an irreducible character of $G$, then Isaacs has defined an associated pair $(W, \gamma)$, called a nucleus of $\chi$. The nucleus is the last term in a certain chain of pairs $(I, \psi)$, where $I$ is a subgroup of $G$ and $\psi$ is an irreducible character of $I$. The length of this chain is an invariant of $\chi$ that we call the nuclear length. In this paper we study bounds on the nuclear length of $\chi$ as a function of the $\pi$-length of $G$ and as a function of the character degree $\chi(1)$.
\end{abstract}

1991 Mathematics subject classification (Amer. Math. Soc.): $20 \mathrm{C} 15$.

\section{Introduction}

If $\pi$ is a set of primes, then the character theory of $\pi$-separable groups demonstrates a remarkable richness that is not shared by finite groups in general. For example, in [1] Gajendragadkar defined the set $\mathfrak{X}_{\pi}(G)$ of $\pi$-special characters of $G$ and showed that if $\alpha$ is $\pi$-special and $\beta$ is $\pi^{\prime}$-special, then $\alpha \beta$ is irreducible. Because a product of characters is so rarely irreducible, this result seems quite surprising. The idea of factoring characters into a product of $\pi$-special and $\pi^{\prime}$-special parts was exploited by Isaacs, who defined a set of irreducible characters $B_{\pi}(G)$ containing the $\pi$-special characters [2]. When $\pi=p^{\prime}$, the complement of a single prime $p$, the characters in $B_{p^{\prime}}(G)$ provide canonical lifts for the irreducible Brauer characters of $G$. Thus the set $\operatorname{IBr}(G)$ of irreducible Brauer characters of $G$ is obtained simply by restricting the characters in $B_{p^{\prime}}(G)$ to the $p$-regular elements of $G$. More generally, the characters in $B_{\pi^{\prime}}(G)$ act as a $\pi$-analogue of Brauer characters, and Slattery has used them to develop a theory of $\pi$-blocks in $\pi$-separable groups (see $[4,5,6]$ ). More recently, Isaacs and Navarro have defined weights and vertices for characters and have proven a $\pi$-analogue of Alperin's Weight Conjecture for $\pi$-separable groups [3].

(C) 1994 Australian Mathematical Society 0263-6115/94 \$A2.00+0.00 
The point of this paper is to return to Isaacs' definition of $B_{\pi}(G)$ in [2] and study it more closely. If $\chi \in \operatorname{Irr}(G)$, then Isaacs constructs a subgroup $I$ and a character $\psi \in \operatorname{Irr}(I)$ with $\psi^{G}=\chi$. The pair $(I, \psi)$ is called a standard inducing pair, and $(I, \psi)=(G, \chi)$ if and only if $\chi$ factors as the product of a $\pi$-special and a $\pi^{\prime}$-special character. One can also construct a standard inducing pair for $\psi$, and this process leads inductively to a pair $(W, \gamma)$ such that $\gamma^{G}=\chi$ and $\gamma$ is the product of a $\pi$-special and a $\pi^{\prime}$-special character. The pair $(W, \gamma)$ is called a nucleus for $\chi$ and is unique up to $G$-conjugacy. Isaacs defines the set $B_{\pi}(G)$ to consist of those irreducible characters $\chi$ for which there is a nucleus $(W, \gamma)$ such that $\gamma$ is itself $\pi$-special.

But it is easy to see that this construction produces more than just the conjugacy class of pairs $(W, \gamma)$. In particular, associated to the character $\chi \in \operatorname{Irr}(G)$ is a nonnegative integer given by the number of times one must apply the inductive procedure before obtaining a nucleus for $\chi$. We call this number the nuclear length of $\chi$, and we write it as $\operatorname{nl}(\chi)$. The purpose of this paper is to study the invariant given by the nuclear length. We will see that it is related to both the character degree $\chi(1)$ and the $\pi$-length $\ell_{\pi}(G)$ of $G$. Specifically, we will prove the following result.

THEOREM A. Let $G$ be a $\pi$-separable group, and let $\chi \in \operatorname{Irr}(G)$. Then $\operatorname{nl}(\chi)$ is bounded by both of the following numbers:

(1) $2 \ell_{\pi}(G)$.

(2) The number of prime divisors (counting multiplicities) of $\chi(1)$.

In fact, the bound given in (2) is a very easy consequence of the definition of the nucleus. When $\chi \in B_{\pi^{\prime}}(G)$, however, we can get somewhat better bounds, and in this case neither bound seems to follow directly from the definitions.

THEOREM B. Let $G$ be a $\pi$-separable group, and let $\chi \in B_{\pi^{\prime}}(G)$. Then $\mathrm{nl}(\chi)$ is bounded by both of the following numbers:

(1) $\ell_{\pi}(G)$.

(2) The number of prime divisors (counting multiplicities) of $\chi(1)_{\pi}$.

Here $\chi(1)_{\pi}$ denotes the $\pi$-part of $\chi(1)$.

Section 2 is devoted to a summary of relevant definitions and results from [1, 2], leading to a precise definition of the nuclear length. Theorems A and B are proven in Section 3.

\section{The Character Theory of $\pi$-Separable Groups}

The purpose of this section is to define the nuclear length and to give a brief review of the relevant aspects of character theory for $\pi$-separable groups. The results of 
interest here come from the work of Gajendragadkar [1] and Isaacs [2]. We assume throughout the section that $\pi$ is a set of primes and $G$ is a $\pi$-separable group.

Recall that if $\chi$ is a character of $G$, then the determinantal order $o(\chi)$ is the order of det $\chi$ in the group of linear characters of $G$. This concept forms the basis for the following definition from [1].

Definition 2.1. Let $G$ be a $\pi$-separable group. A character $\chi \in \operatorname{Irr}(G)$ is said to be $\pi$-special if the following conditions are satisfied:

(1) $\chi(1)$ is a $\pi$-number.

(2) If $S$ is any subnormal subgroup of $G$ and $\sigma$ is any irreducible constituent of $\chi_{S}$, then the determinantal order $o(\sigma)$ is a $\pi$-number.

The set of $\pi$-special characters of $G$ is denoted by $\mathfrak{X}_{\pi}(G)$.

The following result is almost an immediate consequence of the above definition. It appears as [1, Proposition 4.1].

PROPOSITION 2.2. Let $G$ be a $\pi$-separable group, and let $\chi \in \operatorname{Irr}(G)$ be $\pi$-special. If $S \triangleleft \triangleleft G$ and $\sigma \in \operatorname{Irr}(S)$ is a constituent of $\chi_{s}$, then $\sigma$ is $\pi$-special.

PROPOSITION 2.3. Let $G$ be a $\pi$-separable group, and let $N$ be a normal subgroup of $G$ such that $G / N$ is a $\pi$-group. If $\eta \in \operatorname{Irr}(N)$ is $\pi$-special, then every irreducible constituent of $\eta^{G}$ is $\pi$-special.

Proof. See [1, Proposition 4.5].

PROPOSITION 2.4. Let $G$ be a $\pi$-separable group, and let $N$ be a normal subgroup of $G$ such that $G / N$ is a $\pi^{\prime}$-group.

(1) If $\chi \in \operatorname{Irr}(G)$ is $\pi$-special, then $\chi_{N}$ is irreducible and $\pi$-special.

(2) If $\eta \in \operatorname{Irr}(N)$ is $\pi$-special and $G$-invariant, then $\eta$ has a $\pi$-special extension $\hat{\eta} \in \operatorname{Irr}(G)$. Moreover, $\hat{\eta}$ is the only irreducible constituent of $\eta^{G}$ that is $\pi$-special.

ProOF. See [1, Proposition 4.3].

The next result, which appears as Proposition 7.1 of [1], shows that $\pi$-special characters have a remarkable multiplicative property.

PROPOSITION 2.5. Let $G$ be a $\pi$-separable group. If $\alpha \in \mathfrak{X}_{\pi}(G)$ and $\beta \in \mathfrak{X}_{\pi^{\prime}}(G)$, then $\alpha \beta$ is irreducible. Moreover, if $\alpha^{\prime} \in \mathfrak{X}_{\pi}(G)$ and $\beta^{\prime} \in \mathfrak{X}_{\pi^{\prime}}(G)$ satisfy $\alpha \beta=\alpha^{\prime} \beta^{\prime}$, then $\alpha=\alpha^{\prime}$ and $\beta=\beta^{\prime}$.

As in [2], we will say that a character $\chi \in \operatorname{Irr}(G)$ is $\pi$-factorable if it can be written as a product $\alpha \beta$ with $\alpha \in \mathfrak{X}_{\pi}(G)$ and $\beta \in \mathfrak{X}_{\pi^{\prime}}(G)$. The following result, which is an easy consequence of Propositions 2.2 and 2.5, is given as Corollary 2.6 of [2]. 
COROLlaRY 2.6. Let $G$ be a $\pi$-separable group, and let $\chi \in \operatorname{Irr}(G)$ be $\pi$ factorable. If $S \triangleleft \triangleleft G$, then every irreducible constituent of $\chi_{S}$ is $\pi$-factorable.

The main point of [2] is to use the concept of $\pi$-factorability as the basis for defining a certain subset $B_{\pi}(G)$ of $\operatorname{Irr}(G)$ containing the $\pi$-special characters. Because the definitions involved in the construction of $B_{\pi}(G)$ are very important to the work presented in the next section, we recall the construction in detail.

Consider the set $\mathscr{P}$ of all pairs $(H, \theta)$, where $H$ is a subgroup of $G$ and $\theta \in \operatorname{Irr}(H)$. If $(K, \varphi) \in \mathscr{P}$, then we write $(H, \theta) \leq(K, \varphi)$ if $H \subseteq K$ and $\theta$ is a constituent of $\varphi_{H}$. If $g \in G$, let $(H, \theta)^{g}=\left(H^{g}, \theta^{g}\right)$. Then $\leq$ is a partial order on $\mathscr{P}$, and the conjugation action of $G$ respects the partial order. We will write $I_{G}(H, \theta)$ for the stabilizer of $(H, \theta)$ in $G$.

A pair $(S, \sigma)$ is called a $\pi$-factorable subnormal pair if $S \triangleleft \triangleleft G$ and $\sigma$ is $\pi$ factorable. The set of $\pi$-factorable subnormal pairs in $G$ is denoted by $\mathscr{F}(G)$, and we write $\mathscr{F}^{*}(G)$ for the set of maximal elements of $\mathscr{F}(G)$. The following proposition describes a fundamental property of the partial order on $\mathscr{F}(G)$.

PROPOSITION 2.7. Let $G$ be a $\pi$-separable group, and let $\chi \in \operatorname{Irr}(G)$. Then there is a pair $(S, \sigma) \in \mathscr{F}^{*}(G)$ with $(S, \sigma) \leq(G, \chi)$. Moreover, if $(U, \varphi) \in \mathscr{F}(G)$ with $(U, \varphi) \leq(G, \chi)$, then $(U, \varphi)^{g} \leq(S, \sigma)$ for some $g \in G$.

ProOF. See [2, Theorem 3.2].

PROPOSITION 2.8. Let $G$ be a $\pi$-separable group, let $(S, \sigma) \in \mathscr{F}^{*}(G)$, and set $I=I_{G}(S, \sigma)$. Then induction defines a bijection $\operatorname{Irr}(I \mid \sigma) \rightarrow \operatorname{Irr}(G \mid \sigma)$. Moreover, if $S<G$, then $I<G$.

ProOF. See [2, Theorem 4.4 and Lemma 4.5].

We are now ready to present Isaacs' definition of the nucleus of a character. Suppose that $G$ is $\pi$-separable, and let $\chi$ be any irreducible character of $G$. By Proposition 2.7 there is a pair $(S, \sigma) \in \mathscr{F}^{*}(G)$ with $(S, \sigma) \leq(G, \chi)$. Let $I=I_{G}(S, \sigma)$. Then Proposition 2.8 implies that there is a unique character $\psi \in \operatorname{Irr}(I \mid \sigma)$ such that $\psi^{G}=\chi$. In this way we associate a pair $(I, \psi)$ with the original pair $(G, \chi)$, and $(I, \psi)$ is unique up to $G$-conjugacy. The pair $(I, \psi)$ is called a standard inducing pair for $\chi$. If $\chi$ is $\pi$-factorable, then $S=G$ so that $(I, \psi)=(G, \chi)$. But if $\chi$ is not $\pi$-factorable, then $S<G$ and hence $I<G$ by Proposition 2.8. We can then repeat the process by finding a standard inducing pair for $\psi$. Proceeding by induction, we see that there is a chain

$$
(G, \chi)=\left(I_{0}, \psi_{0}\right)>\left(I_{1}, \psi_{1}\right)>\cdots>\left(I_{n}, \psi_{n}\right)=(W, \gamma)
$$

such that $\left(I_{j}, \psi_{j}\right)$ is a standard inducing pair for $\psi_{j-1}$ and $\gamma$ is $\pi$-factorable. Any such pair $(W, \gamma)$ is called a nucleus for $\chi$, and the set of all nuclei for $\chi$ is denoted nuc $(\chi)$. 
Thus nuc $(\chi)$ consists of a single $G$-conjugacy class of pairs, and if $(W, \gamma)$ is a nucleus for $\chi$, then $\gamma^{G}=\chi$. Finally, $B_{\pi}(G)$ denotes the set of all $\chi \in \operatorname{Irr}(G)$ such that there is a pair $(W, \gamma) \in \operatorname{nuc}(\chi)$ with $\gamma \in \mathfrak{X}_{\pi}(G)$. Note that if $(I, \psi)$ is a standard inducing pair for $\chi$, then nuc $(\psi) \subseteq \operatorname{nuc}(\chi)$. Thus $\chi \in B_{\pi}(G)$ if and only if $\psi \in B_{\pi}(I)$.

To reach the main subject of this paper, we now need only one simple observation about Isaacs' definition of the nucleus. Let $\chi \in \operatorname{Irr}(G)$. Because the standard inducing pair $(I, \psi)$ is unique up to $G$-conjugacy, so is the pair $(W, \gamma)$. But we can say more: in fact, the entire chain of pairs from $(G, \chi)$ to $(W, \gamma)$ is unique up to $G$-conjugacy. In particular, the length of the chain is uniquely determined by $\chi$. This observation suggests the following definition.

DEFINITION 2.9. Let $G$ be a $\pi$-separable group, and let $\chi \in \operatorname{Irr}(G)$. Let $(W, \gamma) \in$ $\operatorname{nuc}(\chi)$, and let

$$
(G, \chi)=\left(I_{0}, \psi_{0}\right)>\left(I_{1}, \psi_{1}\right)>\cdots>\left(I_{n}, \psi_{n}\right)=(W, \gamma)
$$

be a chain such that $\left(I_{j}, \psi_{j}\right)$ is a standard inducing pair for $\psi_{j-1}$. Then we say that $\chi$ has nuclear length $n$. We write $\operatorname{nl}(\chi)$ for the nuclear length of $\chi$.

The nuclear length can be regarded as a number that measures how close $\chi$ is to being $\pi$-factorable. Indeed, it is immediate from the definition that $\chi$ is $\pi$-factorable if and only if $\mathrm{nl}(\chi)=0$.

We end this section with a few more results that will be needed to study nuclear lengths in the next section. Because it seems to be convenient for our purposes to focus on $B_{\pi^{\prime}}(G)$ instead of $B_{\pi}(G)$, we have stated the results below for this set of characters.

PROPOSITION 2.10. Let $G$ be a $\pi$-separable group, and let $N$ be a normal subgroup of $G$. Suppose that $\eta \in \operatorname{Irr}(N)$.

(1) Assume that $G / N$ is a $\pi^{\prime}$-group and $\chi \in \operatorname{Irr}(G \mid \eta)$. Then $\eta \in B_{\pi^{\prime}}(N)$ if and only if $\chi \in B_{\pi^{\prime}}(G)$.

(2) Assume that $G / N$ is a $\pi$-group. Then $\eta \in B_{\pi^{\prime}}(N)$ if and only if there exists a character $\chi \in B_{\pi^{\prime}}(G)$ lying over $\eta$. Moreover, in this case $\chi$ is the unique irreducible constituent of $\eta^{G}$ in $B_{\pi^{\prime}}(G)$.

PROOF. See [2, Theorems 6.2 and 7.1].

The following proposition is essentially proven in [2, Theorem 6.2 and Proposition 7.3]. Because not all parts are explicitly stated there, we have sketched a proof for the convenience of the reader. 
PROPOSITION 2.11. Let $G$ be a $\pi$-separable group and $N \triangleleft G$ such that $G / N$ is a $\pi$-group. Let $\eta \in \operatorname{Irr}(N)$, and let $\chi \in \operatorname{Irr}(G \mid \eta)$. Suppose that $(U, \varphi) \in \mathscr{F}^{*}(N)$ with $(U, \varphi) \leq(N, \eta)$ and $(S, \sigma) \in \mathscr{F}^{*}(G)$ with $(U, \varphi) \leq(S, \sigma) \leq(G, \chi)$. Let $(J, \xi)$ and $(I, \psi)$ be the corresponding standard inducing pairs for $\eta$ and for $\chi$ respectively. Then

(1) $U=S \cap N$.

(2) I normalizes $J$.

(3) $I \cap J \triangleleft I J$.

(4) $I /(I \cap J)$ and $J /(I \cap J)$ are $\pi$-groups.

Moreover, if $\chi \in B_{\pi^{\prime}}(G)$, then

(5) $I=I_{G}(U, \varphi)$ and $J=I \cap N$.

(6) $\psi$ lies over $\xi$.

PROOF. The fact that $U=S \cap N$ is [2, Lemma 3.3]. Moreover, [2, Lemma 6.1 and Proposition 7.3] imply that $I$ normalizes $J, I \cap J \triangleleft I J,|I: I \cap J|$ divides $|G: N|^{2}$, and $|J: I \cap J|$ divides $|G: N|$. Hence $I /(I \cap J)$ and $J /(I \cap J)$ are $\pi$-groups.

Now assume that $\chi \in B_{\pi^{\prime}}(G)$. Then $\sigma$ is $\pi^{\prime}$-special by [2, Lemma 5.2], and $S / U$ is a $\pi$-group because it is isomorphic to a subgroup of $G / N$. Thus Proposition 2.4 implies that $\sigma_{U}$ is irreducible and $\pi^{\prime}$-special, so it follows that $\sigma_{U}=\varphi$. Moreover, $U=S \cap N \triangleleft I$ and $I$ stabilizes $\sigma_{U}=\varphi$, so $I \subseteq I_{G}(U, \varphi)$. By [2, Lemma 6.1] we know that $I_{G}(U, \varphi)$ normalizes $S$. But $I_{G}(U, \varphi)$ must also stabilize $\sigma$ because $\sigma$ is the unique $\pi^{\prime}$-special extension of $\varphi$ by Proposition 2.4. Hence $I=I_{G}(U, \varphi)$, and $J=I \cap N$.

Now let $\xi_{0}$ be an irreducible constituent of $\psi_{J}$ lying over $\varphi$, and set $\eta_{0}=\xi_{0}^{N}$. Then Proposition 2.8 implies that $\eta_{0}$ is an irreducible constituent of $\chi_{N}$, so $\eta_{0}^{x}=\eta$ for some $x \in G$. Then $(U, \varphi)^{x} \leq(N, \eta)$ and $(U, \varphi)^{x} \in \mathscr{F}^{*}(N)$, so Proposition 2.7 shows that there is an $n \in N$ with $(U, \varphi)^{x n}=(U, \varphi)$. It follows that $x n \in I$, so we may assume without loss of generality that $x \in I$. Then $\xi_{0}^{x} \in \operatorname{Irr}(J \mid \varphi)$ and $\left(\xi_{0}^{x}\right)^{N}=\eta=\xi^{N}$, so Proposition 2.8 implies that $\xi_{0}^{x}=\xi$. Hence $\xi$ is a constituent of $\psi_{J}$, and (6) follows. This completes the proof.

\section{Bounding the Nuclear Length}

In this section we study the nuclear length of a character $\chi \in \operatorname{Irr}(G)$ and obtain the bounds given in Theorems A and B. Because the strategy is to proceed by induction on the order of the group $G$, we begin by considering a normal subgroup $N$ of $G$, and we try to relate the nuclear length of $\chi$ to that of an irreducible constituent of $\chi_{N}$. The first step in this direction is a technical lemma. 
LEMMA 3.1. Let $G$ be a $\pi$-separable group, and let $N$ be a normal subgroup such that $G / N$ is either a $\pi$-group or a $\pi^{\prime}$-group. Let $\eta \in \operatorname{Irr}(N)$, and let $\chi \in \operatorname{Irr}(G \mid \eta)$. Let $(U, \varphi) \in \mathscr{F}^{*}(N)$ with $(U, \varphi) \leq(N, \eta)$, and let $(J, \xi)$ be the corresponding standard inducing pair for $\eta$. Then there is a pair $(S, \sigma) \in \mathscr{F}^{*}(G)$ with $(U, \varphi) \leq(S, \sigma) \leq$ $(G, \chi)$ such that if $(I, \psi)$ is the corresponding standard inducing pair for $\chi$, then $\left(\psi_{I \cap J}, \xi_{I \cap J}\right) \neq 0$.

PROOF. It suffices to consider the case in which $G / N$ is a $\pi$-group. We prove the result by induction on $\operatorname{nl}(\chi)$. If $\operatorname{nl}(\chi)=0$, then $\chi$ and $\eta$ are both $\pi$-factorable by Corollary 2.6. Hence $(J, \xi)=(N, \eta)$ and $(I, \psi)=(G, \chi)$, so the conclusion of the lemma is satisfied.

Now assume that $\mathrm{nl}(\chi)>0$ so that $\chi$ is not $\pi$-factorable. Choose $(S, \sigma) \in \mathscr{F}^{*}(G)$ with $(U, \varphi) \leq(S, \sigma) \leq(G, \chi)$, and let $(I, \psi)$ be the standard inducing pair for $\chi$ determined by $(S, \sigma)$. Then Proposition 2.11 implies that $I$ normalizes $J, I \cap J \triangleleft I J$, and $I /(I \cap J)$ and $J /(I \cap J)$ are $\pi$-groups.

Let $\theta=\psi^{I J}$, and let $\xi_{0} \in \operatorname{Irr}(J \mid \varphi)$ be a constituent of $\theta_{J}$. Then $\xi_{0}^{N} \in \operatorname{Irr}(N)$ by Proposition 2.8, and $\chi$ lies over $\xi_{0}^{N}$ because it lies over $\xi_{0}$. Hence $\xi_{0}^{N}=\eta^{x}$ for some $x \in G$. It follows that $(U, \varphi) \leq\left(N, \eta^{x}\right)$, so $(U, \varphi)^{x^{-1}} \leq(N, \eta)$ and $(U, \varphi)^{x^{-1}} \in \mathscr{F}^{*}(N)$. Thus there is an $n \in N$ such that $(U, \varphi)^{x^{-1}}=(U, \varphi)^{n}$, and $n x \in I_{G}(U, \varphi)$. Hence we may assume that $x \in I_{G}(U, \varphi)$ so that $x$ normalizes $J$. Moreover, $\xi^{x}$ lies over $\varphi^{x}=\varphi$ and $\left(\xi^{x}\right)^{N}=\left(\xi^{N}\right)^{x}=\eta^{x}=\xi_{0}^{N}$, so $\xi^{x}=\xi_{0}$ by Proposition 2.8. Thus $\left(J, \xi^{x}\right) \leq(I J, \theta)$, and $(J, \xi) \leq(I J, \theta)^{x^{-1}}$.

If we now replace $(S, \sigma)$ by $(S, \sigma)^{x^{-1}}$, then $(I, \psi)$ is replaced by $(I, \psi)^{x^{-1}}$ and $(I J, \theta)$ by $(I J, \theta)^{x^{-1}}$. Hence we may assume that $(J, \xi) \leq(I J, \theta)$. Then $\xi$ is a constituent of $\theta_{J}=\left(\psi^{I J}\right)_{J}=\left(\psi_{I \cap J}\right)^{J}$, so there is a character $\zeta \in \operatorname{Irr}(I \cap J)$ that is a constituent of both $\psi_{I \cap J}$ and $\xi_{I \cap J}$. This completes the proof.

LEMMA 3.2. Let $G$ be a $\pi$-separable group, and let $N$ be a normal subgroup of $G$ such that $G / N$ is either a $\pi$-group or a $\pi^{\prime}$-group. Let $\eta \in \operatorname{Irr}(N)$, and suppose that there is a subgroup $U \triangleleft \triangleleft N$ such that $(U, \varphi) \in \operatorname{nuc}(\eta)$. If $\chi \in \operatorname{Irr}(G \mid \eta)$, then $\mathrm{nl}(\chi) \leq 1$.

PROOF. It suffices to consider the case in which $G / N$ is a $\pi$-group. Let $(S, \sigma) \in$ $\mathscr{F}^{*}(G)$ with $(U, \varphi) \leq(S, \sigma) \leq(G, \chi)$, and let $(I, \psi)$ be the standard inducing pair for $\chi$ determined by $(S, \sigma)$. Since $U \triangleleft \triangleleft N$ and $(U, \varphi) \in \operatorname{nuc}(\eta)$, it follows that $(U, \varphi) \in \mathscr{F}^{*}(N)$ and $I_{N}(U, \varphi)=U$. Then Proposition 2.11 shows that $U \triangleleft I$ and $I / U$ is a $\pi$-group, so $I / S$ is a $\pi$-group.

Write $\sigma=\alpha \beta$, where $\alpha \in \mathfrak{X}_{\pi}(S)$ and $\beta \in \mathfrak{X}_{\pi^{\prime}}(S)$. Then $\beta$ is invariant in $I$ and $|I: S|$ is a $\pi$-number, so $\beta$ extends to $\hat{\beta} \in \mathfrak{X}_{\pi^{\prime}}(I)$ by Proposition 2.4. Hence $\sigma^{I}=(\alpha \beta)^{I}=\alpha^{I} \hat{\beta}$. All irreducible constituents of $\alpha^{I}$ are $\pi$-special by Proposition 2.3, 
so all irreducible constituents of $\sigma^{I}$ are $\pi$-factorable. In particular, $\psi$ is $\pi$-factorable, and $\operatorname{nl}(\chi) \leq 1$.

PROPOSITION 3.3. Let $G$ be a $\pi$-separable group, and let $N, H_{1}$, and $H_{2}$ be subgroups of $G$ such that $N \triangleleft H_{1}$ and $N \triangleleft H_{2}$. Suppose that $H_{1} / N$ and $H_{2} / N$ are $\pi$-groups. Let $\eta \in \operatorname{Irr}(N)$, and let $\chi_{1} \in \operatorname{Irr}\left(H_{1} \mid \eta\right)$ and $\chi_{2} \in \operatorname{Irr}\left(H_{2} \mid \eta\right)$. Then $\left|\mathrm{nl}\left(\chi_{1}\right)-\operatorname{nl}\left(\chi_{2}\right)\right| \leq 1$.

PROOF. We prove the result by induction on $\operatorname{nl}\left(\chi_{1}\right)$. If $\mathrm{nl}\left(\chi_{1}\right)=0$, then $\chi_{1}$ and $\eta$ are $\pi$-factorable by Corollary 2.6. Hence $(N, \eta) \in$ nuc $(\eta)$, and Lemma 3.2 shows that $\mathrm{nl}\left(\chi_{2}\right) \leq 1$. Thus $\left|\mathrm{nl}\left(\chi_{1}\right)-\mathrm{nl}\left(\chi_{2}\right)\right| \leq 1$ in this case. Similarly, we get the desired result if $\mathrm{nl}\left(\chi_{2}\right)=0$.

Thus we may assume that $\mathrm{nl}\left(\chi_{1}\right)>0$ and $\mathrm{nl}\left(\chi_{2}\right)>0$. Let $(U, \varphi) \in \mathscr{F}^{*}(N)$ with $(U, \varphi) \leq(N, \eta)$, and let $(J, \xi)$ be the corresponding standard inducing pair for $\eta$. For $i=1,2$ Lemma 3.1 implies that there is a pair $\left(S_{i}, \sigma_{i}\right) \in \mathscr{F}^{*}\left(H_{i}\right)$ with $(U, \varphi) \leq\left(S_{i}, \sigma_{i}\right) \leq\left(H_{i}, \chi_{i}\right)$ such that if $\left(I_{i}, \psi_{i}\right)$ is the corresponding standard inducing pair for $\chi_{i}$, then $\left(\left(\psi_{i}\right)_{I_{i} \cap J}, \xi_{l_{i} \cap J}\right) \neq 0$. Moreover, Proposition 2.11 shows that $I_{i} \cap J \triangleleft I_{i} J$, and $I_{i} /\left(I_{i} \cap J\right)$ and $J /\left(I_{i} \cap J\right)$ are $\pi$-groups. In particular, it follows that $O^{\pi}\left(I_{i}\right)=O^{\pi}\left(I_{i} \cap J\right)=O^{\pi}(J)$ for $i=1$, 2. Set $M=O^{\pi}(J)$. Let $\theta_{1} \in \operatorname{Irr}(M)$ be a common constituent of $\left(\psi_{1}\right)_{M}$ and $\xi_{M}$, and let $\theta_{2} \in \operatorname{Irr}(M)$ be a common constituent of $\left(\psi_{2}\right)_{M}$ and $\xi_{M}$. Then there is an $x \in J$ such that $\theta_{1}=\theta_{2}^{x}$. If we replace $\left(H_{2}, \chi_{2}\right)$ by $\left(H_{2}, \chi_{2}\right)^{x}$ and $\left(S_{2}, \sigma_{2}\right)$ by $\left(S_{2}, \sigma_{2}\right)^{x}$, then we may assume that $\theta_{1}=\theta_{2}$.

Since $M=O^{\pi}\left(I_{1}\right)=O^{\pi}\left(I_{2}\right)$, we know that $M \triangleleft I_{1}$ and $M \triangleleft I_{2}$, and $I_{1} / M$ and $I_{2} / M$ are $\pi$-groups. Moreover, $\psi_{1} \in \operatorname{Irr}\left(I_{1} \mid \theta_{1}\right)$ and $\psi_{2} \in \operatorname{Irr}\left(I_{2} \mid \theta_{1}\right)$. Thus it follows by induction that

$$
\left|\mathrm{nl}\left(\chi_{1}\right)-\operatorname{nl}\left(\chi_{2}\right)\right|=\left|\mathrm{nl}\left(\psi_{1}\right)-\operatorname{nl}\left(\psi_{2}\right)\right| \leq 1,
$$

and this completes the proof.

We will use Proposition 3.3 in the following form.

COROLLARY 3.4. Let $G$ be a $\pi$-separable group, and let $N$ be a normal subgroup of $G$ such that $G / N$ is either a $\pi$-group or a $\pi^{\prime}$-group. If $\eta \in \operatorname{Irr}(N)$ and $\chi \in \operatorname{Irr}(G \mid \eta)$, then $\mathrm{nl}(\chi) \leq 1+\mathrm{nl}(\eta)$.

PROOF. It suffices to consider the case in which $G / N$ is a $\pi$-group. The result then follows by applying Proposition 3.3 with $H_{1}=G, H_{2}=N, \chi_{1}=\chi$, and $\chi_{2}=\eta$.

THEOREM 3.5. Let $G \neq 1$ be a $\pi$-separable group, and let $\chi \in \operatorname{Irr}(G)$. Let $1=G_{0} \triangleleft G_{1} \triangleleft \cdots \triangleleft G_{n}=G$ be any chain of subgroups such that $G_{i} / G_{i-1}$ is either a $\pi$-group or a $\pi^{\prime}$-group for $1 \leq i \leq n$. Then $\operatorname{nl}(\chi) \leq n-1$. 
PROOF. We prove the result by induction on $n$. If $n=1$, then $G$ is either a $\pi$-group or a $\pi^{\prime}$-group, so $\mathrm{nl}(\chi)=0=n-1$.

Now assume that $n>1$, and let $\eta \in \operatorname{Irr}\left(G_{n-1}\right)$ be a constituent of $\chi_{G_{n-1}}$. Then Corollary 3.4 shows that $\mathrm{nl}(\chi) \leq 1+\mathrm{nl}(\eta)$. Moreover, we know by induction that $\mathrm{nl}(\eta) \leq n-2$, so it follows that $\mathrm{nl}(\chi) \leq n-1$.

We now obtain Theorem A as an easy consequence of this result.

PROOF OF THEOREM A. To prove (1), consider the chain of subgroups

$$
1 \triangleleft O_{\pi^{\prime}}(G) \triangleleft O_{\pi^{\prime} \pi}(G) \triangleleft O_{\pi^{\prime} \pi \pi^{\prime}}(G) \triangleleft \cdots \triangleleft G .
$$

The number of successive quotients in this series that are $\pi$-groups is $\ell_{\pi}(G)$, and the number that are $\pi^{\prime}$-groups is at most $\ell_{\pi}(G)+1$. Thus the total number of quotients in the series is at most $2 \ell_{\pi}(G)+1$, and Theorem 3.5 implies that $\mathrm{nl}(\chi) \leq 2 \ell_{\pi}(G)$.

To prove (2), we merely use the definition of the nucleus. If $(W, \gamma) \in \operatorname{nuc}(\chi)$, then there is a chain

$$
(W, \gamma)=\left(I_{n}, \psi_{n}\right)<\left(I_{n-1}, \psi_{n-1}\right)<\cdots<\left(I_{0}, \psi_{0}\right)=(G, \chi)
$$

in which $\left(I_{j}, \psi_{j}\right)$ is a standard inducing pair for $\psi_{j-1}$. Since $W=I_{n}<I_{n-1}<\cdots<$ $I_{0}=G$, we see that $n=\operatorname{nl}(\chi)$ is at most the number of prime divisors (counting multiplicities) of $|G: W|$. Because $|G: W|$ divides $\gamma^{G}(1)=\chi(1)$, the result follows.

We now turn our attention to characters in $B_{\pi^{\prime}}(G)$. The assumption that $\chi \in B_{\pi^{\prime}}(G)$ allows us to obtain better bounds on $\mathrm{nl}(\chi)$ with rather little additional effort. Indeed, the following proposition already improves upon the conclusion of Corollary 3.4.

PROPOSITION 3.6. Let $G$ be a $\pi$-separable group, and let $N$ be a normal subgroup such that $G / N$ is a $\pi^{\prime}$-group. Let $\chi \in B_{\pi^{\prime}}(G)$, and let $\eta$ be an irreducible constituent of $\chi_{N}$. Then $\operatorname{nl}(\chi)=\operatorname{nl}(\eta)$.

PROOF. We prove the result by induction on $|G|$. If $\mathrm{nl}(\chi)=0$, then $\chi \in B_{\pi^{\prime}}(G)$ is $\pi$-factorable and hence $\chi$ is $\pi^{\prime}$-special. Then $\eta$ is also $\pi^{\prime}$-special by Proposition 2.2, so $\mathrm{nl}(\eta)=0$. Conversely, suppose that $\mathrm{nl}(\eta)=0$. Because $\eta \in B_{\pi^{\prime}}(N)$ by Proposition 2.10, it follows that $\eta$ is $\pi^{\prime}$-special. Then Proposition 2.3 implies that $\chi$ is $\pi^{\prime}$-special, so $\operatorname{nl}(\chi)=0$.

Now suppose that $\mathrm{nl}(\chi)>0$ so that $\mathrm{nl}(\eta)>0$ as well. Let $(U, \varphi) \in \mathscr{F}^{*}(N)$ with $(U, \varphi) \leq(N, \eta)$, and let $(J, \xi)$ be the corresponding standard inducing pair for $\eta$. Then by Lemma 3.1 there is a pair $(S, \sigma) \in \mathscr{F}^{*}(G)$ with $(U, \varphi) \leq(S, \sigma) \leq(G, \chi)$ such that if $(I, \psi)$ is the corresponding standard inducing pair for $\chi$, then $\left(\psi_{I \cap J}, \xi_{I \cap J}\right) \neq 0$. Let $\zeta \in \operatorname{Irr}(I \cap J)$ be a constituent of both $\psi_{I \cap J}$ and $\xi_{I \cap J}$. Because $\operatorname{nl}(\chi)>0$ 
and $\mathrm{nl}(\eta)>0$, we have $I<G$ and $J<N \subseteq G$. Moreover, Proposition 2.11 shows that $I$ normalizes $J, I \cap J \triangleleft I J$, and $I /(I \cap J)$ and $J /(I \cap J)$ are both $\pi^{\prime}$-groups. Thus by induction it follows that $\mathrm{nl}(\psi)=\operatorname{nl}(\zeta)$ and $\mathrm{nl}(\xi)=\operatorname{nl}(\zeta)$. Hence $\mathrm{nl}(\chi)=1+\operatorname{nl}(\psi)=1+\mathrm{nl}(\xi)=\mathrm{nl}(\eta)$, and this completes the proof.

PROPOSITION 3.7. Let $G$ be a $\pi$-separable group and $N$ a normal subgroup of $G$ such that $G / N$ is a $\pi$-group. Let $\chi \in B_{\pi^{\prime}}(G)$, and let $\eta$ be an irreducible constituent of $\chi_{N}$. If $\eta$ is $G$-invariant, then $\operatorname{nl}(\chi)=\operatorname{nl}(\eta)$.

PROOF. We prove the result by induction on $\mathrm{nl}(\eta)$. If $\mathrm{nl}(\eta)=0$, then $\eta$ is $\pi^{\prime}$-special because $\eta \in B_{\pi^{\prime}}(G)$ by Proposition 2.10. Since $\eta$ is $G$-invariant by assumption, Proposition 2.4 shows that $\eta$ has a unique $\pi^{\prime}$-special extension $\hat{\eta} \in \operatorname{Irr}(G)$. In particular, $\hat{\eta} \in B_{\pi^{\prime}}(G)$. Thus $\chi=\hat{\eta}$ by Proposition 2.10, and $\mathrm{nl}(\chi)=0$.

Now assume that $\mathrm{nl}(\eta) \geq 1$. Choose $(U, \varphi) \in \mathscr{F}^{*}(N)$ with $(U, \varphi) \leq(N, \eta)$ and $(S, \sigma) \in \mathscr{F}^{*}(G)$ with $(U, \varphi) \leq(S, \sigma) \leq(G, \chi)$. Let $(J, \xi)$ and $(I, \psi)$ be the corresponding standard inducing pairs for $\eta$ and for $\chi$ respectively. Then Proposition 2.11 shows that $U=S \cap N, J=I \cap N, I=I_{G}(U, \varphi)$, and $\psi$ lies over $\xi$. Hence $I / J$ is a $\pi$-group. Moreover, if $x \in I$, then $x$ stabilizes $(U, \varphi)$ and $(N, \eta)$, and $x$ normalizes $J$. Hence $x$ must stabilize $\xi$, and we see that $\xi$ is $I$-invariant. Thus it follows by induction that $\mathrm{nl}(\chi)=\mathrm{nl}(\psi)+1=\operatorname{nl}(\xi)+1=\operatorname{nl}(\eta)$, as desired.

THEOREM 3.8. Let $G$ be a $\pi$-separable group and $\chi \in B_{\pi^{\prime}}(G)$. Let $1=G_{0} \triangleleft G_{1} \triangleleft$ $\cdots \triangleleft G_{n}=G$ be a chain of subgroups such that $G_{i} / G_{i-1}$ is either a $\pi$-group or a $\pi^{\prime}$-group for $1 \leq i \leq n$. Choose a corresponding chain $\left(G_{0}, \chi_{0}\right) \leq\left(G_{1}, \chi_{1}\right) \leq \cdots \leq$ $\left(G_{n}, \chi_{n}\right)=(G, \chi)$. Then $\mathrm{nl}(\chi)$ is at most the number of indices $i$ with $1 \leq i \leq n$ for which the following two conditions are satisfied:

(1) $G_{i} / G_{i-1}$ is a $\pi$-group.

(2) $\chi_{i-1}$ is not $G_{i}$-invariant.

PROOF. We prove the result by induction on $n$. If $n \leq 1$, then $G$ is either a $\pi$-group or a $\pi^{\prime}$-group, $\operatorname{sonl}(\chi)=0$.

Now suppose that $n>1$. For $1 \leq m \leq n$ let $f(m)$ be the number of indices $i$ with $1 \leq i \leq m$ such that (1) and (2) hold. Then we know by induction that $\operatorname{nl}\left(\chi_{n-1}\right) \leq$ $f(n-1)$. If $G_{n} / G_{n-1}$ is a $\pi^{\prime}$-group, then $f(n-1)=f(n)$ and $\operatorname{nl}(\chi)=\operatorname{nl}\left(\chi_{n-1}\right)$ by Proposition 3.6. Similarly, when $G_{n} / G_{n-1}$ is a $\pi$-group and $\chi_{n-1}$ is $G$-invariant, it follows that $f(n-1)=f(n)$ and $\operatorname{nl}(\chi)=\operatorname{nl}\left(\chi_{n-1}\right)$ by Proposition 3.7. Thus we may assume that $G_{n} / G_{n-1}$ is a $\pi$-group and $\chi_{n-1}$ is not $G$-invariant. In this case $f(n)=f(n-1)+1$ and $\mathrm{nl}(\chi) \leq \mathrm{nl}\left(\chi_{n-1}\right)+1$ by Corollary 3.4 , so we again conclude that $\mathrm{nl}(\chi) \leq f(n)$.

Finally, we can now prove Theorem B. 
PROOF OF THEOREM B. The bound given in (1) follows by applying Theorem 3.8 to the chain of subgroups

$$
1 \triangleleft O_{\pi^{\prime}}(G) \triangleleft O_{\pi^{\prime} \pi}(G) \triangleleft O_{\pi^{\prime} \pi \pi^{\prime}}(G) \triangleleft \cdots \triangleleft G .
$$

We prove by induction on $|G|$ that the number described in (2) also bounds $\mathrm{nl}(\chi)$. Let $N$ be a maximal normal subgroup of $G$, and let $\eta \in \operatorname{Irr}(N)$ be a constituent of $\chi_{N}$. Then there is an integer $e \geq 1$ such that

$$
\chi(1)=e\left|G: I_{G}(\eta)\right| \eta(1),
$$

and $e$ divides $|G: N|$. If $G / N$ is a $\pi^{\prime}$-group, then $\mathrm{nl}(\chi)=\mathrm{nl}(\eta)$ by Proposition 3.6. But $\chi(1)_{\pi}=\eta(1)_{\pi}$, so the result follows by induction.

Now suppose that $G / N$ is a $\pi$-group. Then $\operatorname{nl}(\chi) \leq \operatorname{nl}(\eta)+1$ by Corollary 3.4, and $\eta(1)_{\pi}$ divides $\chi(1)_{\pi}$, so it suffices by induction to show that if $\operatorname{nl}(\chi)=\operatorname{nl}(\eta)+1$, then $\chi(1)_{\pi} \neq \eta(1)_{\pi}$. But we know by Proposition 3.7 that if $\mathrm{nl}(\chi)=\mathrm{nl}(\eta)+1$, then $\eta$ is not $G$-invariant. Hence $\chi(1)_{\pi}=e\left|G: I_{G}(\eta)\right| \eta(1)_{\pi} \neq \eta(1)_{\pi}$, and this completes the proof.

It would be interesting to know whether the bounds on $\mathrm{nl}(\chi)$ given in Theorems $\mathrm{A}$ and $B$ are the best possible in any reasonable sense. In the case of Theorem B, for instance, it is easy to find individual examples of a $\pi$-separable group $G$ with a character $\chi \in B_{\pi^{\prime}}(G)$ such that $\operatorname{nl}(\chi)=\ell_{\pi}(G)$. But for every nonnegative integer $n$ we can ask whether there is a $\pi$-separable group $G$ of $\pi$-length $n$ and a character $\chi \in B_{\pi^{\prime}}(G)$ of nuclear length $n$. This question, along with the analogous questions for the other bounds on $\mathrm{nl}(\chi)$, seems to be difficult to answer.

\section{References}

[1] D. Gajendragadkar, 'A characteristic class of characters of finite $\pi$-separable groups', J. Algebra 59 (1979), 237-259.

[2] I. M. Isaacs, 'Characters of $\pi$-separable groups', J. Algebra 86 (1984), 98-128.

[3] I. M. Isaacs and G. Navarro, 'Weights and vertices for characters of $\pi$-separable groups', preprint.

[4] M. Slattery, 'Pi-blocks of pi-separable groups, I', J. Algebra 102 (1986), 60-77.

[5] — 'Pi-blocks of pi-separable groups, II', J. Algebra 124 (1989), 236-269.

[6] — ' 'Pi-blocks of pi-separable groups, III', J. Algebra 158 (1993), 268-278.

School of Mathematical Sciences

Australian National University

Canberra ACT 0200

Australia

(e-mail: wheeler@pell.anu.edu.au) 